\title{
Prevención en organización de personas jurídicas, principio del daño y mitigación del lavado de activos
}

\author{
Marlene B. Malqui Falcón \\ bmalqui@hotmail.com \\ Escuela de Posgrado UNFV
}

\section{RESUMEN}

El presente estudio se corresponde con lo propuesto en la tesis universitaria destinada a obtener el grado académico de Doctor en Derecho, en donde se procuró identificar el nivel de incidencia entre la implementación del modelo de prevención sobre responsabilidad administrativa de la organización de las personas jurídicas, orientado bajo el principio del daño, frente a la comisión, por detección y mitigación, del lavado de activos (Lima, 2020); desarrollándose, a nivel metodológico, una indagación científica correlacional, con enfoque cuantitativo y diseño no experimental, aplicando a los Abogados los instrumentos de recojo de datos. Así, el estudio permitió arribar a la principal conclusión de que entre la implementación del modelo de prevención sobre responsabilidad administrativa de la organización de las personas jurídicas tiene una relación asociativa simple con la detección y mitigación del lavado de activos, con una correlación de tipo inversa y con un nivel de incidencia negativa, pues el valor numérico es de -0,684 (bilateral). Así, la temática en comento no ha incidido de forma positiva en la detección y mitigación delictiva, por diferentes aspectos como la voluntariedad de la implementación del modelo de prevención por parte de las personas jurídicas en su estructura organización y comercial; dicha implementación debe encuadrase al principio del daño, que deviene en equilibrar la relación entre la individualidad y la sociedad, lo que hace posible que el ser humano asuma su rol actuante dentro de éste mecanismo.

Palabras clave: modelo de prevención; responsabilidad administrativa; personas jurídicas; principio de daño; lavado de activos. 


\title{
Preventión in organization of legal persons, pinciple of damage and mitigation of money laundering
}

\begin{abstract}
The objective of this study corresponds to what was proposed in the university thesis that allowed us to obtain the academic degree of Doctor of Law, where it was sought to identify the level of incidence between the implementation of the prevention model on administrative responsibility of the organization of legal persons, oriented under the principle of harm, against the commission, by detection and mitigation, of money laundering (Lima, 2020); To achieve this, a correlational scientific inquiry was developed at a methodological level, with a quantitative approach and a nonexperimental design, having as a study population a group of legal professionals to whom the data collection instruments were applied. in the sample quantity obtained. Thus, the study made it possible to reach the main conclusion that the implementation of the prevention model on administrative responsibility of the organization of legal entities has a simple associative relationship with the detection and mitigation of money laundering, with an inverse correlation and with a negative incidence level, since the numerical value is -0.684 (bilateral). This means that the issue in question has not had a positive impact on the detection and mitigation of money laundering, due to different aspects such as the voluntary nature of the implementation of the prevention model by legal entities in their organizational and commercial structure; This implementation must respond to or be framed by the principle of damage, a principle that results in balancing the relationship between individuality and society, which makes it possible for the human being, as an individual, to assume their acting role within this mechanism.
\end{abstract}

Keywords: prevention model; administrative responsibility; legal entities; principle of damage; money laundering.

Artículo recibido: 02 Setiembre. 2021 Aceptado para publicación: 30 Setiembre. 2021

Correspondencia: bmalqui@ hotmail.com Conflictos de Interés: Ninguna que declarar 


\section{INTRODUCCIÓN}

En atención a los modelos de prevención sobre responsabilidad administrativa, a implementarse en la organización de las personas jurídicas, para la detección y mitigación del lavado de activos, la normativa nacional ha dejado en claro que su implementación en la estructura organizacional y comercial de la persona jurídica es voluntaria; agregando que la misma buscará prevenir, detectar y mitigar la comisión de delitos, promoviendo la integridad y transparencia en su gestión, siendo ésta una de las razones de su problemática, puesto que quedado bajo el ámbito de la discrecionalidad de las mismas personas jurídicas; es por ello que se pone a debate respecto a si la intervención de la Superintendencia de Mercado de Valores (SMV) constituye o no un requisito de procedibilidad para el ejercicio de la acción penal por parte del Ministerio Público, ya que por mandato de ésta norma legal se requiere, como paso previo, de la emisión de un Informe Técnico referido al análisis sobre la implementación del modelo de prevención, el cual resulta determinante, incluso para el archivo de las investigaciones fiscales.

La presente investigación, desde su diseño, se formuló como problema general la interrogante siguiente: ¿Cuál es el nivel de incidencia entre la implementación del modelo de prevención sobre responsabilidad administrativa de la organización de las personas jurídicas orientado bajo el principio del daño frente a la detección y mitigación del lavado de activos (Lima, 2020)?; en igual sentido, fueron formulados dos problemas específicos, con la siguiente redacción: a) ¿Qué relación existe entre la regulación del Informe de la Superintendencia del Mercado de Valores (SMV) con la restricción a la facultad del Ministerio Público para investigar de manera autónoma el delito de lavado de activos (Lima, 2020)?; y, b) ¿Qué relación existe entre las competencias normativas innatas de la Superintendencia de Mercado de Valores y las competencias asignadas a la Unidad de Inteligencia Financiera para evitar duplicidades de esfuerzos y contradicciones entre entidades (Lima, 2020)?.

Frente a ello fue necesario plantearnos el objetivo general que ya ha sido señalado al inicio del presente artículo, lo cual nos conllevó a la elaboración de los objetivos específicos, siendo éstos redactados de la siguiente manera: a) Precisar la relación existente entre la regulación del Informe de la Superintendencia del Mercado de Valores (SMV) y la restricción a la facultad del Ministerio Público para investigar de manera autónoma el delito de lavado de activos; y, b) Examinar la relación existente entre las competencias normativas innatas de la Superintendencia de Mercado de Valores y las competencias asignadas a la Unidad de Inteligencia Financiera para evitar duplicidad de esfuerzos y contradicciones entre entidades. El planteamiento metodológico que antecede 
ameritó la formulación de la siguiente hipótesis: Entre la implementación del modelo de prevención sobre responsabilidad administrativa de la organización de las personas jurídicas orientado bajo el principio del daño y la comisión del lavado de activos, por detección y mitigación, existe una relación asociativa simple de tipo inversa con un nivel de incidencia negativa (Lima, 2020), la cual ha sido materia de contrastación con los resultados para confirmar su validación, conjuntamente con las siguientes hipótesis específicas: a) Entre la regulación del Informe de la Superintendencia del Mercado de Valores (SMV) y la restricción a la facultad del Ministerio Público para investigar de manera autónoma el delito de lavado de activos se espera encontrar una relación asociativa simple de tipo inverso con un nivel de incidencia negativa (Lima, 2020); y, b) Entre las competencias normativas innatas de la SMV y las competencias asignadas a la Unidad de Inteligencia Financiera para evitar duplicidad de esfuerzos y contradicciones entre entidades se espera encontrar una relación asociativa simple de tipo directa con un nivel de incidencia positiva (Lima, 2020).

Para llegar a la respectiva contrastación, según la proyección del trabajo inicial, se plantearon las siguientes variables principales: Variable X: Implementación del modelo de prevención sobre responsabilidad administrativa de la organización de las personas jurídicas orientado bajo el principio del daño; y, Variable Y: Detección y mitigación del lavado de activos; siendo que, para su medición, se plantearon las dimensiones de cada variable. Así la variable $\mathrm{X}$ fue dimensionada en torno a la regulación del Informe de la Superintendencia del Mercado de Valores y asignación de competencias normativas innatas de la Superintendencia de Mercado de Valores; por su parte, la variable Y se dimensionó en Restricción a la facultad del Ministerio Público para investigar de manera autónoma el delito de lavado de activos y asignación de competencias de la Unidad de Inteligencia Financiera para evitar duplicidad de esfuerzos y contradicciones entre entidades, dichas dimensiones conllevaron a la identificación de sus indicadores que coadyuvaron, de manera efectiva, a la medición de las variables y dimensiones para alcanzar los objetivos propuestos y realizar la contrastación de hipótesis.

Considerando el supuesto que no hay dogmas, que en el ámbito filosófico, no puedan ser objeto de críticas y reformulaciones teóricas que pretende alcanzar nuestra indagación, es que da a luz este artículo que aborda un tópico de significativa utilidad práctica en la esfera social, introduciéndose entre las últimas tendencias de cohabitación social, cuyo rasgo relevante es el dechado de interconexión subjetiva en un rango global, tanto a nivel de los hechos efectuados en la realidad, como aquellos elementos pertenecientes a la esfera intelectual; lo anteriormente expuesto, 
fundamentado por la idea central de que todo sujeto, objeto o idea existente es susceptible de análisis, alteración, comprobación, revisión, ampliación o eliminación.

Es debido al avance teórico, científico y tecnológico que, en la actualidad, existe una variedad de conexiones de carácter comercial e inversiones dinerarias que se mantienen en progresiva formación y expansión (principalmente la amplia gama de tecnologías de la información). En tal sentido, la tecnología y la globalización se identifican en este siglo XXI, como factores cardinales del orden social, no obstante, debemos señalar que, a pesar de los impactos positivos que han resultado de estos factores, también existen efectos negativos, como lo es el empleo de estos instrumentos con la finalidad de delinquir. Definitivamente, esta situación pone en alerta a los gobiernos, pues no se puede depender de los métodos tradicionales para hacerle frente a la criminalidad, sino que, debe establecerse un sistema moderno que pueda combatir esta problemática de forma óptima.

Siendo así, con el objetivo de solucionar distintos defectos producidos por la poca claridad y ambigüedades teóricas, se debe hacer uso de la filosofía; en vista que, los avances tecnológicos y científicos; la globalización de los mercados y su virtualización; el progreso en el ámbito de comunicaciones y transportes; conllevan el crecimiento positivo de la totalidad de los países del globo. Sin embargo, no ha existido profilaxis de nuestra parte, con respecto de los diversos problemas que podrían aparecer, topándonos con la universalización del crimen organizado, por medio de diversos convenios delictivos entre organización que se ubican en territorios distintos, abarcando no solo el plano nacional sino también trascendiendo a este. De esta forma, la criminalidad se constituye como una organización de estructura compleja y variada que pretenden obtener abundante capital que los enriquece y consecuentemente, les facilita seguir efectuando sus múltiples operaciones, la comisión de nuevos ilícitos y el disfrute del dinero, generado a partir de los ilícitos efectuados.

Entonces, como se ha explicado en el párrafo anterior, nos orientamos a brindar una solución viable - que, debido al constante cambio de las cosas, no es el remedio definitivo - más bien, se constituye como una contribución que pretende explicar de forma más detallada los conceptos medulares del tópico que refiere a la persona jurídica y la responsabilidad que deriva de esta en la ciencia del derecho.

Ahora, en la mística área de la filosofía, ha existido una oposición entre dos corrientes: Iusnaturalismo y Positivismo; cuyo resultado derivó en la noción contemporánea del derecho, siendo un vínculo de índole factual y moral, idea absolutamente relevante para nuestra investigación, con respecto a la elección de la postura que esté más adecuada y coordinada con la 
medida de las variables de la indagación. En ese sentido, durante esta época contemporánea, el vínculo presente en la escala mundial, nos hace concluir que nuestra especie se dirige a unificar progresivamente el orden económico, siendo este de carácter universal y único. Es por ello, que la permanencia de todo tipo de comportamientos criminales efectuados por organizaciones delictivas transnacionales genera efectos nocivos para las distintas economías en la esfera mundial que, se encuentran alteradas debido a la introducción de capitales ilícitos en ellas, con el objetivo de camuflarlos como dinero legal y no ser identificados como dinero de fuente ilícita.

Evidentemente esta problemática, incide negativamente en el orden económico de las naciones, toda vez que origina competencia desleal cuando no vulnera de forma directa a los privados que efectúan distintas operaciones y actos en el marco de la legalidad y formalidad, mientras que esta organización lo pasa por alto y saca ventajas a costo de la ilicitud, entre otros supuestos que se efectúan en los diversos países.

De hecho, las naciones toman decisiones múltiples para combatir esta grave cuestión referida a los comportamientos criminales y organizados que trascienden a jurisdicción de los países; por ello, es que aparece un novedoso tipo penal denominado "lavado de activos". Esta fue la razón principal para que la ONU tome la decisión de establecer diversas estrategias y medidas para prever y sancionar la criminalidad contemporánea, orientándose a conminar de manera estricta la fuente ilícita de capitales empleados por las organizaciones delictivas. Sin embargo, no es hasta la Convención de Viena de 1988 que, este ilícito penal se materializa como figura delictiva.

Conjuntamente a lo anteriormente indicado, aparecen regulaciones nuevas de índole mundial con miras a combatir la delincuencia transnacional. De tal manera que, en virtud del Convenio de Palermo del 2000, se tipifican comportamientos autónomos de riesgo que, únicamente por el hecho de desplegar tal conducta, ya fundamenta la conminación de carácter penal, por haber encajado en el supuesto de conformar o ser parte de alguna organización delictiva. Dicho convenio hizo hincapié principalmente en las industrias o empresas delictivas.

Es recién con la Convención de Mérida (2003) que la Convención de las Naciones Unidas contra la Corrupción pone más atención al contexto criminal que se estaba desarrollando a nivel internacional, tomando una perspectiva de análisis más meticulosa, al examinarse a más detalle las intervenciones que realizan aquellos sujetos activos que forman parte de las estructuras de poder, con ello se busca delimitar e identificar cuáles son serían los posibles funcionarios o servidores públicos que están inmersos en el crimen organizado, independientemente si se tratase de integrantes propiamente dicho, algún colaborar o dirigente. 
Se puede afirmar entonces, que esta convención mundial dio como resultado la realización de diversas acciones políticas, sociales, jurídicas, que buscan luchar contra la corrupción funcional.

Años después, cuando se realizó la Cumbre de las Naciones mundiales, se puso en tela de discusión que existían personas jurídicas que apoyaban a la criminalidad organizada, especialmente al ilícito de lavado de activos, por lo cual, mediante esta modalidad criminal se hacía pasar dinero, bienes, ganancias que procedían de crímenes, como si fuera obtenidos legalmente acorde al ordenamiento jurídico. Ante esto, se produjeron una serie de recomendaciones direccionadas a regular la responsabilidad jurídica, tanto de empresas, como de industrias criminales que se dedican a realizar actos ilícitos, pudiendo ser tanto de carácter civil, penal o administrativo, siendo independiente de las regulaciones de las personas naturales.

Luego de analizar este contexto histórico internacional, y ya adentrándonos en un plano más local, pero tomando en cuenta el análisis normativo que recomienda las Naciones Unidas, en el Perú se ha ido creando modelos y estrategias para prevenir y reprimir la delincuencia moderna que tanto daño hace al país.

Basta recordar que, con la promulgación del Código Penal de 1991, los legisladores peruanos acertadamente a través del art. $105^{\circ}$ del cuerpo normativo en mención, en su Parte General, habían prescrito una serie de consecuencias de carácter accesorio con la finalidad de que las personas jurídicas se desliguen de realizar conductas delictivas.

Pero lamentablemente, en lugar de discutir la naturaleza jurídica propia de esta institución, en relación a precisar si realmente hay un merecimiento de una sanción penal o no, en base a un sistema vicarial o de responsabilidad conjunta (el cual precisa que debe de aplicarse previamente la sanción de la persona natural que hizo provecho de este), son hasta la actualidad pocos los casos que terminen en una sentencia condenatoria; es por ello que, este tema es de mucho interés y produce polémica, que van a permitir una constantes revaluación de lo que está regulado.

No es de sorprenderse entonces, que los legisladores peruanos optarán por una estrategia políticocriminal por la cual se incluya directamente en los tipos penales que correspondiesen, la presencia de personas jurídicas como, por ejemplo, en los delitos aduaneros, tributarios o de lavado de activos. Sin embargo, esta regulación específica pecó de inoficiosa, en el sentido que no se llegó a constatar que se hubiesen sancionado a las personas jurídicas, por lo cual estos sujetos de derecho se siguieron beneficiando y ayudando a la realización del lavado de dinero.

Por otro lado, al tener la intención el Perú de poder formar parte de la Organización de Cooperación de Desarrollo Económico (OCDE), trajo como consecuencia una serie de beneficios, entre ellas, el 
de cumplir con sus exigencias en relación a reprimir efectivamente a las personas jurídicas que estuviesen involucradas en la comisión de actos delincuenciales, dando mayor importancia a aquellos organismos criminales que tengan como integrantes a funcionarios públicos.

Ante esto, mediante la Comisión Nacional Anticorrupción (CAN) se plantearon varios proyectos, en la que resalta la responsabilidad penal directa de las personas jurídicas relacionadas al delito de cohecho activo transnacional. El sector empresarial, sin embargo, planteó la responsabilidad administrativa a las empresas, lo que generó dos ámbitos de regulación jurídica,

Como iban las cosas, y al estar el Perú con la necesidad de formar parte de la OCDE, se reguló de manera específica la intervención de las personas jurídicas que están involucradas en actos delictivos.

Los dispositivos de autorregulación o modelos de prevención de cumplimiento normativo (los llamados Compliance Programs), bajo una especie de controles sociales informales, no habían venido cumpliendo su fin concreto, el cual era prevenir el involucramiento de las empresas o industrias en hechos delictuosos. Como consecuencia, la Ley $\mathrm{N}^{\circ} 30424$, del 21 de abril de 2016, reguló la responsabilidad administrativa de las personas jurídicas por el delito de cohecho activo transnacional, cuya entrada en vigencia estaba prevista, según la Primera Disposición Complementaria Final, para el 1 de julio de 2017.

Lamentablemente, dicha cuerpo legal presentaba diversas inconsistencias, empezando que se encontraba delimitado a un solo delito. Además, pese a ser interpretado como una responsabilidad administrativa, su regulación y contenido era puramente de carácter penal. Agregado a ello, la aplicación resulta compleja al darse una serie de eximentes de responsabilidad, atenuantes o la suspensión de la ejecución, por pretender seguir el modelo de prevención. Hecho que hace surgir las siguientes cuestiones: ¿En qué consiste el modelo de prevención que las personas jurídicas han de implementar en sus organizaciones?; ¿Cuáles son las conductas delictivas más recurrentes que se pueden cometer a través de los entes organizacionales?; ¿Qué incidencia logra tener el modelo de prevención en las empresas respecto de la comisión de delitos cometidos por entes organizacionales?, entre otras interrogantes que nos llevarán a conocer los resultados efectivos generados a partir de las nuevas reglas jurídicas existentes en nuestro país.

Al referirse a las personas jurídicas, autores como Atehortúa (2005) y Alcalde (2015) señalan que una elaboración conceptual resulta ser abstracta y compleja; a pesar de ello, autores como Belaunde (2010) refiere que se trata de centros de incriminación normativa, para que la sociedad se organice en su actividad económica y para llevar a cabo fines que la legislación reconozca su propia legalidad. 
No deja de tener razón Da Mota (2005) cuando refiere que viene a ser la entidad formada por una compañía de personas “a las cuales la norma jurídica le atribuye una legalidad jurídica” (p. 269). En este punto, ha de quedar claro que de acuerdo a la Ley $\mathrm{N}^{\circ} 30424$, las personas jurídicas podían ser acreedoras de sanciones administrativas a pesar de no ser personas naturales; en tal sentido, en la doctrina nacional tenemos a Caro (2017) y en la extranjera tenemos a Mila (2020) quienes sostiene que en la doctrina del derecho penal se hace referencia al postulado "societas delinquere non potest", que está relacionado con la afirmación que las sociedades no pueden delinquir; sin embargo, esto no es del todo cierto puesto que la ley mencionada líneas arriba señala la existencia de una "responsabilidad administrativa", la cual no se realiza en dicha esfera sino en un proceso penal con sus garantías y regido por su normativa adjetiva.

El Estado establece parámetros políticos criminales para contrarrestar el fenómeno criminal económico denominado lavado de activos, pero tales políticas no solo se dan en el ámbito penal que imponen sanciones de forma posterior a la comisión del acto delictivo; sino también el ámbito administrativo debido a que prevé herramientas para prever la comisión de lavado de activos. En tal sentido, Del Carpio Delgado (1997) deja notar que resulta de necesidad realizar, en todo lo posible, tanto la prevención como el control administrativo en lo que respecta a la gestión de los riesgos de la elaboración del lavado de activos o de su favorecimiento, ello "con el fin de facilitar la delimitación de los contornos extrapenales del riesgo permitido en estos ámbitos vulnerables” (p. 166).

Ahora bien, haciendo referencia al conceptual del trabajo de investigación, tenemos que Del Cid Gómez (2007) y Perotti (2009) dejan en claro que por el lavado de activos se busca ocultar, esconder, impedir o disfrazar la condición ilegal o ilícita de los activos. En el mismo sentido, se tiene a Barral (2003) para quien el lavado de activos, como proceso criminal, está orientado a disfrazar el real origen de la procedencia de los bienes o sus efectos para que no se le vinculen con actos delictivos precedentes; de allí que, lo que se busca disimular la ilegitimidad del capital producto de un previo que viene a ser acto delictivo. Sin embargo, Suarez (1996) sostiene que el delito de lavado de activos no entraña un injusto en sí mismo sino un injusto referido al delito previo.

Sobre el lavado de activos, los autores Fabián (2007) y Gálvez (2004) sostiene que desde una perspectiva terminológica, tanto el blanqueo, el reciclaje, el lavado, la normalización, la legalización, como la reconversión de bienes, están referidos al proceso que busca utilizar en actividades legales un determinado patrimonio proveniente de actividades ilegales. Por su parte, autores nacionales como Mendoza (2017) y extranjeros como Ponce, Piedrahita y Villagómez 
(2019) señalan que "la terminología "blanqueo" se corresponde con la expresión francesa blanchimented'argent o blanchiment de capitanx, y la portuguesa "branqueamento de capitais". Para la Real Academia Española, el blanqueo de capitales viene a ser el delito consistente en adquirir o comerciar con bienes, particularmente dinero, procedentes de la comisión de un delito grave.

Así, resulta ser que el lavado de activos ha de ser entendido como el conglomerado de operaciones de naturaleza financiera y comercial que "buscan la introducción, al fujo económico legal de un país, de un conjunto de bines, recursos y capitales que se originaron directa o indirectamente de una actuación ilícita" (Prado, 2007, p. 96).

En ese sentido, los autores como Anaya, Trejo y Fernández (2008) y De la Haza, Aguedo y Rosales (2018) resaltan que las empresas del sector financiero forman parte del proceso en la etapa de prevención y detección mediante sus procedimientos orientados a adoptar políticas internas para evitar filtraciones de casos de lavado en los sistemas operativos. Ahora bien, estando a la intervención de las personas jurídicas en el delito de lavado de activos, y su obligación de combatirlo, el jurista peruano García (2017) expresa que:

En el conglomerado de parámetros de buen gobierno empresarial, se puede enfatizar que es necesario la incorporación dentro de la entidad empresarial una cultura de cumplimiento que impida la inobservancia de la normales legales por intermedio de las medidas de prevención y detección (Compliance). Es así que, dichas medidas son una garantía para los sujetos que deseen invertir o quieran ligar sus interés económicos con la gestión de una entidad jurídica, que podría ponerse en peligro porque la entidad empresarial ingrese a una controversia judicial, situación que se pretendía mediante un programa de cumplimiento normativo, lo que tendría incidencia no solo de carácter ético sino también económico, por lo que, el Compliance es un instrumento vital en el buen gobierno de una entidad empresarial. (p. 25)

Por su parte, el modelo de prevención no es sino un esquema preventivo que brinda importantes alternativas de naturaleza técnica y práctica para impulsar el desarrollo y expansión del llamado derecho penal preventivo "o de pertinente contrapeso a la adopción de un modelo de responsabilidad penal propia de la persona jurídica" (Prado, 2016, p. 304). No debe dejarse de lado que "esta criminalidad se produce de modo genérico dentro de empresas, que se da en la partición de compromiso y la partición de capacidades prácticos, donde el beneficio final es la consecuencia de acciones incrementadas de diferentes dependientes" (Reategui, 2016, pp. 1981-1982). 


\section{Así, el estudio realizado nos permite arribar a las conclusiones siguientes:}

1) Como primera conclusión tenemos que entre la implementación del modelo de prevención sobre responsabilidad administrativa de la organización de las personas jurídicas tiene una relación asociativa simple con la detección y mitigación del lavado de activos, con una correlación de tipo inversa con un nivel de incidencia negativa, pues el valor numérico es de -0,684 (bilateral). Por tal motivo se tiene que el implementar el modelo de prevención sobre responsabilidad administrativa de la organización de las personas jurídicas no ha incidido de forma positiva en la detección y mitigación del lavado de activos, por diferentes aspectos como la voluntariedad de la implementación del modelo de prevención por parte de las personas jurídicas en su estructura organización y comercial. Es conveniente resaltar que la incidencia negativa de los modelos de prevención en la detección y mitigación del lavado de activos es en respuesta al alto índice de informalidad que se vive en el país.

2) Como segunda conclusión se tiene que entre la regulación del Informe de la Superintendencia del Mercado de Valores (SMV) y la facultad que tiene el Ministerio Público para investigar de manera autónoma los delitos de lavados de activos existe una relación asociativa simple, dicha a asociación es negativa, pues el valor numérico de -0,712 (bilateral); lo que quiere decir que, la regulación del Informe de la Superintendencia del Mercado de Valores (SMV) repercute de forma inversa y negativa en la facultad del Ministerio Público para investigar de manera autónoma el delito de lavado de activos; es decir, los informes de la Superintendencia del Mercado de Valores ocasionan una mayor restricción de la facultad del Ministerio Público al momento de iniciar la persecución del delito de lavado de activos; todo ello debido a que la intervención de la Superintendencia de Mercado de Valores (SMV) mediante la emisión de un Informe Técnico resulta determinante incluso para el archivo de las investigaciones fiscales, por lo que se manifiesta como una restricción o limitación a la facultad del Ministerio Público para investigar de manera autónoma el delito de lavado de activos.

3) Como última conclusión se sostiene que entre las competencias normativas innatas de la Superintendencia de Mercado de Valores y las competencias asignadas a la Unidad de Inteligencia Financiera existe una relación asociativa simple de tipo inversa con un nivel de incidencia negativa, pues el valor numérico es de - 0,543 (bilateral); por lo que se entiende que las competencias normativas innatas de la Superintendencia de Mercado de Valores repercuten de forma inversa a las competencias asignadas a la Unidad de Inteligencia Financiera; lo que quiere decir que se ocasiona una duplicidad de esfuerzos y contradicciones entre dichas 
entidades mencionadas. La referida situación se origina porque conforme a lo regulado en la Segunda Disposición Complementaria del Reglamento de la Ley $\mathrm{N}^{\circ} 30424$, se otorga la competencia a la Superintendencia de Mercados de Valores para efectuar el Informe Técnico con miras a la comisión del delito de lavado de activos; sin embargo, teniendo en cuenta que existe una entidad especializada denominada Unidad de Inteligencia Financiera, la misma que por sus competencias es la que mejor se encontraría llamada a la emisión del Informe Técnico, ello en la búsqueda de reducirse esfuerzos y hasta posibles contradicciones entre organismos de apoyo a la función fiscal.

\section{MATERIALES Y MÉTODOS}

Conforme lo dispone la metodología de la investigación, una investigación del tipo correlacional corresponde a una medida sobre la relación asociativa que dos variables o más pudieran tener entre sí; de tal manera que se presente una correlación simple o perfecta y positiva o negativa; siendo tal el tipo de investigación adoptado para el presente estudio. Con respecto al enfoque, se desarrolla bajo el enfoque cualitativo, debido a que se medirá la percepción sobre implementación de modelos de prevención en la regulación de responsabilidad administrativa de las personas jurídicas respecto de su correlación asociativa con la detección y mitigación del lavado de activos; lo que nos lleva a establecer un diseño de investigación no experimental, por ser en última instancia la conducta social la que será objeto de estudio, reafirmando la inexistencia de experimentos sobre los temas objeto de estudio, acudiendo como unidades de análisis a las personas jurídicas cuyo rubro comercial se encuentra dentro de los parámetros de incidencia recurrente en el delito de lavado de activos, respecto del rubro con más alta incidencia, según informe de la entidad competente para este fin. De manera complementaria, se tuvo en cuenta, como unidad de análisis, a los Operadores del Derecho, que en total suman 57,521 abogados en el departamento de Lima, lo cual nos permitió contar con una muestra poblacional de 100 Abogados, acudiendo a la formula estadística estándar de muestreo.

De lo anterior queda claro que una de las técnicas para la recopilación de información que se utilizó en el desarrollo de nuestra investigación fue el fichaje, que permitió selección, sistematizar y clasificar la información sobre los temas de estudio. También se utilizó la encuesta; por tanto, los instrumentos empleados corresponden a la ficha y al cuestionario, respectivamente; en éste último su elaboración recurrió a la Escala de Likert. 


\section{RESULTADOS}

En la obtención de resultados se tiene, respecto a la TABLA 1, que la correcta implementación y adecuado funcionamiento del modelo de prevención es permitido en atención al Informe de la Superintendencia del Mercado de Valores, tal como lo señala el $48^{\circ}$ de los encuestados, a los que se suman el $18 \%$ que están totalmente de acuerdo con lo consultado (ver FIGURA 1). También se tiene que, $76 \%$, de entre aquellos que están de acuerdo y totalmente de acuerdo, con que el modelo de prevención en la regulación de la responsabilidad administrativa de las personas jurídicas deba de estar encuadrada dentro del principio del daño. También se tiene que el $96 \%$ de encuestados está de acuerdo y totalmente de acuerdo en que el modelo de prevención debe responder en áreas específicas de riesgo y contar con un sistema de procedimiento de denuncia. Para ello se tiene que el 97\% es de opinión que el modelo de prevención requiere de etapas de evaluación y monitoreo, tal como se desprende de la TABLA 2, a la que se acompaña la FIGURA 2; en igual sentido, el 77\% está de acuerdo, o muy de acuerdo, en que la Superintendencia de Mercado de Valores deba tener competencias normativas que le sean innatas, y por la cual puede (en igual porcentaje de opiniones) dictar normas legales en materia de mercado o de valores, lo cual hace que tenga la capacidad y competencia para la supervisión del cumplimiento de tales normas, así como la competencia para promover y estudiar el comportamiento de los mercados y los valores, tal como lo señalan el $91 \%$ y $90 \%$ de encuestados, respectivamente. Todo esto referido a la variable $\mathrm{X}$, referida a la implementación de modelos de prevención en la regulación de responsabilidad administrativa de las personas jurídicas.

En lo que respecta a la variable Y, sobre la prevención, detección y mitigación del delito de lavado de activos, se tiene que, si bien se establece legalmente que el Ministerio Público tiene la potestad amplia de disponer, requerir y acusar en casos de delitos de lavado de activos, lo cierto es que en la praxis jurisdiccional los Operadores del Derecho señalan estar en desacuerdo y en muy en desacuerdo con esto, así resulta de la TABLA 3, que refleja así el 57\% y 17\% de opiniones de los encuestados, respectivamente y que es de graficarse en la FIGURA 3. Quedando claro, para el 77\% de encuestados, que el Ministerio Público actúa en interdependencia con otras actividades. Siendo que para el $77 \%$ de operadores del Derecho encuestados están de acuerdo que el Ministerio Público investiga el delito de lavado de activos con interdependencia de otras entidades. Finalmente, el 91\% están de acuerdo y totalmente de acuerdo, en que la Unidad de Investigaciones deberá de estar implementada de los mecanismos, medios y normatividad (respaldada por la respectiva dogmática de incidencia) necesarias para el análisis adecuado de la información para la detección del lavado 
de activos, tal como lo comenta el 91\%; en tanto que el $86 \%$ de los encuestados, finalmente, es de opinión que la Unidad de Inteligencia Financiera debe contar con la competencia en lo que respecta a la trasmisión de información para la detección del lavado de activos; debiéndosele asignar competencia para evitar duplicidad de esfuerzos y contradicciones entre entidades públicas.

\section{DISCUSIÓN}

Respecto a algunos aspectos relevantes del modelo de prevención se ha obtenido que los Abogados de Lima Metropolitana y del Callao están de acuerdo en sostener que el modelo de prevención, en la regulación de la responsabilidad administrativa de las personas jurídicas, debe encuadrarse en el principio del daño; debiendo aquel responder en áreas específicas de riesgo y contar con un sistema de procedimiento de denuncia; por lo que, se requiere de etapas de evaluación y monitoreo, debiendo contar, además, con un procedimiento de denuncia y una eta de evaluación y monitoreo, lo cual presenta una relativa coincidencia con los resultados obtenidos en la investigación llevada a cabo por Amat y Flores (2015) quienes sostienen que la cultura preventiva, en lo que respecta al modelo de gestión de riesgo en los casos de las conductas delictivas enmarcadas en los tipos penales de lavado de activos, debe generar el fortalecimiento de la organización de las respectivas entidades en donde se implementa, de tal forma que el modelo a implementarse sea uno ejecutado bajo criterios de planificación y organización, que pueda responder, claramente, a las propias peculiaridades y características de la entidad o institución jurídica de que se trate.

En base a los resultados generales se procedió a realizar la contrastación de la hipótesis general, obteniendo que la implementación del modelo de prevención sobre responsabilidad administrativa de la organización de las personas jurídicas tiene una relación asociativa simple con la detección y mitigación del lavado de activos, significando que aquella correlación significativa se presenta en un nivel de incidencia negativa, pues el valor numérico es de -0,684 (bilateral); lo que coindice con lo sostenido por Clavijo (2016) quien en sus investigación concluye que al hacer referencia a los diversos mecanismos de prevención y de control, a los que se les ha dado por identificar como nomenclatura Criminal Compliance, que vienen a ser, a su vez, los Programas de Cumplimiento Normativo, se tiene que éstos han devenido, en el transcurrir del tiempo, en idóneos y eficientes frente a lo que representa la lucha contra aquel mal endémico que se viene apoderando de la sociedad y que no encuentra frenos, a pesar de la diversa legislación penal que existe, vale decir a la corrupción y a la propia criminalidad empresarial, la misma que se viene generando y ampliando desde diversas partes del mundo, además de ser pertinente ante la generación de la llamada cultura del cumplimiento y del buen gobierno corporativo a nivel de los diversos tipos de empresas; aún 
con ello, no es menos cierto señalar que éstas consideraciones logran ser rebasadas, contundentemente, por la realidad, en donde se llega a demostrar que, en el caso peruano, su implementación es muy limitada.

\section{REFERENCIAS BIBLIOGRÁFICAS}

Anaya Ayala, J., Trejo, R. y Fernández de Lara, R. (2008). Políticas contra el lavado de dinero aplicables a instituciones de crédito y sociedades financieras de objeto limitado. Boletín mexicano de derecho comparado, 41(121), 13-43.

Alcalde Silva, Jaime. (2015). Notas sobre el concepto de persona jurídica sin fines de lucro a propósito de la Ley 20.845 sobre inclusión escolar. Revista chilena de derecho privado, (25), 315-333

Atehortúa, J. (2005). Dimensión institucional de la persona jurídica en el derecho colombiano. La sociedad y la empresa como institución. Revista de Derecho Privado, (8), 47-91.

Barral, J. (2003). Legitimación de bienes procedentes de la comisión de delito: análisis de la ley 25246 de encubrimiento y lavado de activos de origen ilícito. Ad Hoc, Buenos AiresArgentina.

Belaunde, J. (2010). Comentarios al codigo civil. Tomo I. Lima - Perú: Gaceta Juridica.

Caro Coria, D. (2014). ¿Derecho penal en la era del "Compliance”? Derecho Penal \& Procesal Penal. Delitos de Crimen Organizado y Cuestiones Actuales. Lima: Editorial Grijley.

Da Mota, C. (2005). Teoria geral do Direito Civil (4 ${ }^{\mathrm{a}}$ ed.). Portugal: Coimbra editores

Del Cid Gómez, J. (2007). Blanqueo internacional de capitales: como detectarlo y prevenirlo. Barcelona: Editorial Deusto.

Del Carpio Delgado, J (1997). El delito de blanqueo de bienes en el nuevo Código Penal. Valencia: Tirant lo Blanch.

De La Haza Barrantes, A., Aguedo Huiza, B. y Rosales Vicente, M. (2018). Ni dejar hacer ni dejar pasar: el compromiso de las instituciones bancarias peruanas frente al lavado de activos a través de la implementación de una metodología por riesgo. Derecho PUCP, (80), 281-331.

Fabián Caparros, E. (2008). Corrupción y delincuencia económica. Bogotá: Universidad Santo Tomás.

Gálvez Villegas, T. (2004). El delito de lavado de activo: Criterios penal y procesales penalesAnálisis de la nueva ley 27765. Lima: Editorial Grijley.

García Cavero, P. (2017). CRIMINAL COMPLIANCE. En especial Compliance anticorrupción y anti-lavado de activos. Lima: Pacífico Editores 
Mendoza Llamacponcca, F. (2017). El delito de lavado de activos: Aspectos sustantivos y procesales del tipo base como delito autónomo (1ra. ed.). Lima: Instituto Pacifico.

Mila, Frank. (2020). La responsabilidad penal de las personas jurídicas en el derecho ecuatoriano. Ius et Praxis, 26(1), 149-170

Perotti, J. (2009). La problemática del lavado de dinero y sus efectos globales: una mirada a las iniciativas internacionales y las políticas argentinas. UNISCI Discussion Papers, (20), 7899

Prado Saldarriaga, V. (2016). Consecuencias Jurídicas del Delito. Giro Punitivo y Nuevo Marco Legal. Lima: IDEMSA.

Ponce, A.; Piedrahita, P. y Villagómez, R. (2019). Toma de decisiones y responsabilidad penal frente al lavado de activos en Ecuador. Revista de Política Criminal, 14 (28), 365-384.

Reategui, J. (2016). Tratado de Derecho Penal: Parte general. Lima : Legales Instituto.

Suarez, Carlos. (1996). Blanqueo de capitales y merecimiento de la pena: Consideraciones críticas a la luz de la legislación española. Cedernos de Política Criminal, (58), 125-154. 


\section{ANEXOS}

\section{Tabla 1}

El informe de la Superintendencia del Mercado de Valores.

\begin{tabular}{|c|c|c|c|}
\hline & \multirow{3}{*}{$\begin{array}{l}\text { Respuesta } \\
\text { Totalmente en desacuerdo } \\
\text { Fn desacuerdo }\end{array}$} & \multirow{2}{*}{$\begin{array}{c}\text { Frecuencia } \\
4\end{array}$} & \multirow{2}{*}{$\begin{array}{c}\text { Porcentaje } \\
4 \%\end{array}$} \\
\hline \multirow{5}{*}{$\begin{array}{l}\text { El informe de la } \\
\text { Superintendencia del } \\
\text { Mercado de Valores (SMV) } \\
\text { permitirá verificar la } \\
\text { correcta implementación y } \\
\text { adecuado funcionamiento } \\
\text { del modelo de prevención }\end{array}$} & & & \\
\hline & & 14 & $14 \%$ \\
\hline & Ni de acuerdo ni en desacuerdo & 16 & $16 \%$ \\
\hline & De acuerdo & 48 & $48 \%$ \\
\hline & Totalmente de acuerdo & 18 & $18 \%$ \\
\hline & OTAL & 100 & $100 \%$ \\
\hline
\end{tabular}

Fuente: Trabajo de campo realizado vía Google forms en el mes de mayo de 2021.

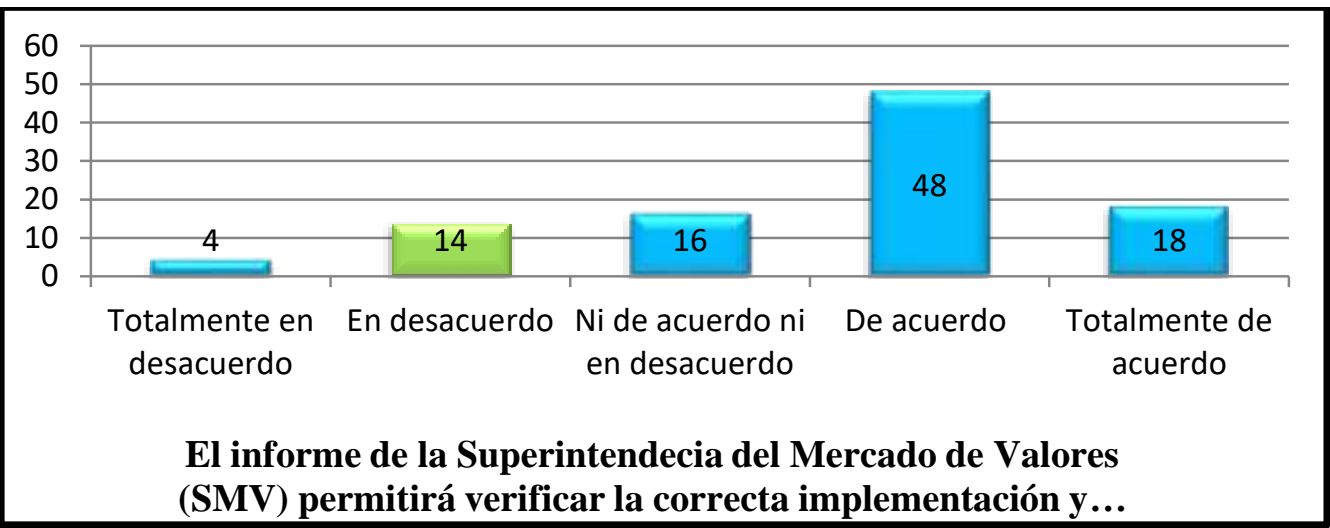

Figura 1. El informe de la Superintendencia del Mercado de Valores

Tabla 2

Etapas de evaluación y monitoreo del modelo de prevención.

\begin{tabular}{llcc}
\hline \multicolumn{1}{c}{ Ítem 4 } & \multicolumn{1}{c}{ Respuesta } & Frecuencia & Porcentaje \\
\hline \multirow{2}{*}{$\begin{array}{l}\text { El modelo de prevención } \\
\text { requiere de etapas de evaluación } \\
\text { y monitoreo. }\end{array}$} & Totalmente en desacuerdo & 3 & $3 \%$ \\
\cline { 2 - 4 } & En desacuerdo & 0 & $0 \%$ \\
\cline { 2 - 4 } & Ni de acuerdo ni en & 0 & $0 \%$ \\
\cline { 2 - 4 } & desacuerdo & 60 & $60 \%$ \\
\cline { 2 - 4 } & De acuerdo & 37 & $37 \%$ \\
\hline & TOTAL & $\mathbf{1 0 0}$ & $\mathbf{1 0 0 \%}$ \\
\hline
\end{tabular}


Fuente: Trabajo de campo realizado vía Google forms en el mes de mayo de 2021.

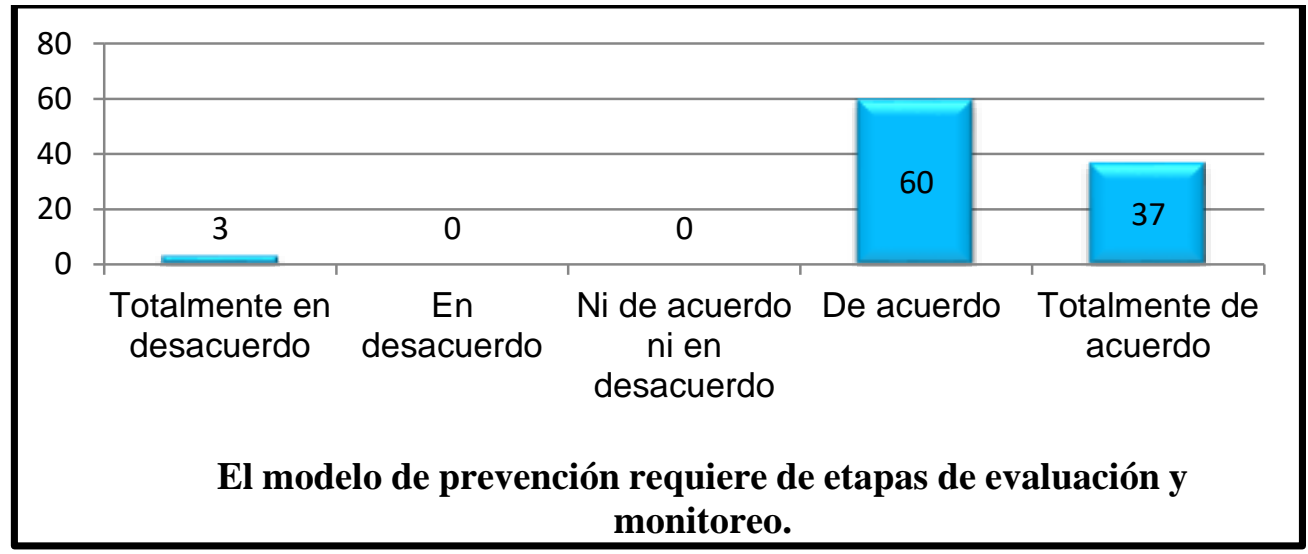

Figura 2. Etapas de evaluación y monitoreo del modelo de prevención.

Tabla 3

Facultades del Ministerio Público en la investigación del lavado de activos.

\begin{tabular}{|c|c|c|c|}
\hline Ítem 1 & Respuesta & Frecuencia & Porcentaje \\
\hline \multirow{5}{*}{$\begin{array}{l}\text { El Ministerio Público ostenta } \\
\text { facultades amplias de disponer, } \\
\text { requerir y acusar en la } \\
\text { investigación del delito de } \\
\text { lavado de activos. }\end{array}$} & Totalmente en desacuerdo & 17 & $17 \%$ \\
\hline & En desacuerdo & 57 & $57 \%$ \\
\hline & $\begin{array}{l}\text { Ni de acuerdo ni en } \\
\text { desacuerdo }\end{array}$ & 7 & $7 \%$ \\
\hline & De acuerdo & 7 & $7 \%$ \\
\hline & Totalmente de acuerdo & 12 & $12 \%$ \\
\hline \multicolumn{2}{|c|}{ TOTAL } & 100 & $100 \%$ \\
\hline
\end{tabular}

Fuente: Trabajo de campo realizado vía Google forms en el mes de mayo de 2021.

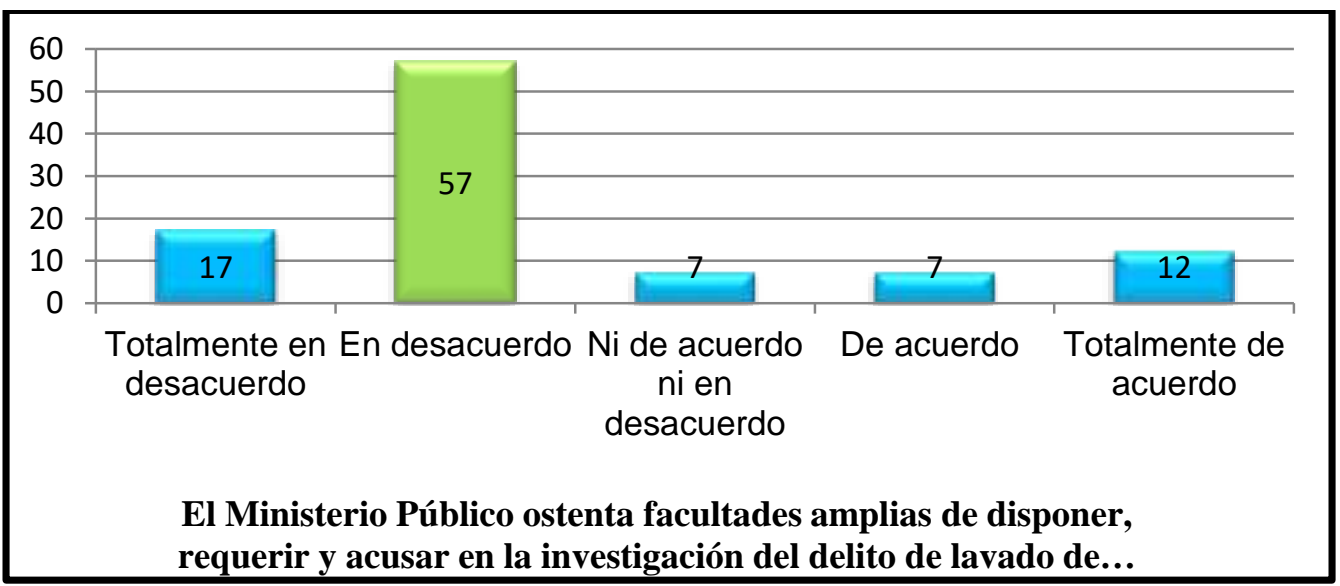

Figura 3 Facultades del Ministerio Público en la investigación del lavado de activos. 